\title{
El proceso de profesionalización del periodista en España o la historia de nunca acabar
}

\author{
Jesús Díaz del Campo Lozano*
}

Recibido: 19 de julio de 2012 Aprobado: 8 de septiembre de 2012

\begin{abstract}
Resumen
El periodismo ha recorrido un largo camino para llegar a ser una profesión. En este artículo se analiza la situación de los periodistas en España, para tratar de averiguar si la actividad periodística ha alcanzado ese estatus.

Existe un alto grado de consenso entre los expertos sobre cuáles son las características principales para poder decir que una ocupación ha llegado a ser una profesión: se trata de una ocupación a tiempo completo, cuenta con un código ético, se necesita formación oficial para ejercerla, una organización y asociación se ha puesto en marcha, etc.
\end{abstract}

El objetivo último es determinar si el periodismo cumple esas características y si, en consecuencia, ha alcanzado el estatus de profesión en España

Palabras clave: periodismo, profesionalismo, enseñanza del periodismo, ética profesional, asociacionismo.

* Este artículo se enmarca en la línea de investigación "La ética periodística en la Unión Europea" que el autor lleva desarrollando varios años y que, en algunas fases de la misma, ha contado con la financiación del Ministerio de Educación y Cultura del Gobierno de España.

** Licenciado y doctor en Periodismo por la Universidad Complutense de Madrid (España). Profesor asociado de la Universidad Internacional de la Rioja (España). Periodista con más de 15 años de experiencia en diversos medios de comunicación. Dirección electrónica: jesus.diaz@unir.net 


\title{
Professionalization Process of the Journalist in Spain or the History with no End
}

\begin{abstract}
Journalism has gone through a long way to become a profession. In this paper, the situation of journalists in Spain is analyzed in order to find if the journalistic activity has reached such status.

There is high consensus among experts about the main characteristics to state that an occupation has become a profession. It is referred to a full-time occupation; it has an ethical code, official formation is necessary to perform it, an organization and an association has started, etc.

The last objective is to determine if journalism meets these requirements and if consequently, it has reached the status of a profession in Spain.

Key words: Journalism, professionalism, teaching of journalism, professional ethics, associationism.
\end{abstract}




\section{Introducción}

El artículo es parte un trabajo más amplio sobre la ética periodística en la Unión Europea (UE), como parte de la cual se consideró necesario definir el estatus del periodismo y del periodista en los diversos países que forman parte de la UE, ya que se estima que es un factor fundamental y, a la vez, íntimamente relacionado con el desarrollo de la autorregulación y de los diversos mecanismos deontológicos en cada uno de ellos.

\section{Metodología}

En consonancia con la metodología de la investigación, se trata de aclarar, en primer lugar, el significado etimológico y las características que definen el término "periodista" para, a continuación, hacer lo mismo con el vocablo "profesión". A partir de ahí, se aplican los rasgos propios de este último a la situación actual del periodismo en España con el objetivo de determinar si se puede inferir que este ha alcanzado el estatus propio de una actividad profesional. A la hora de delimitar el marco conceptual, teniendo en cuenta el amplísimo número de autores que han abordado los procesos de profesionalización, se decidió limitar el análisis a aquellos que lo han hecho desde una perspectiva relacionada de manera directa o indirectamente con el mundo del periodismo.

\section{El periodista, un sujeto difícil de definir}

Intentar definir en qué consiste el periodismo y quién es o quién puede ser considerado periodista no es tarea sencilla. Expresiones como "actividad de contornos imprecisos", "oficio de naturaleza ambigua" o "profesión sin límites" han sido utilizadas a menudo para referirse al ejercicio periodístico. Además, en el fondo de estas cuestiones subyace otra incógnita que constituye probablemente el debate fundamental y para el cual no se ha alcanzado por ahora una solución definitiva: si el periodismo constituye o no una profesión.
Una de las pruebas más evidentes de la dificultad de llegar a una definición precisa se produjo en España durante la comparecencia de 14 personalidades del periodismo ante la Comisión Cons titucional del Congreso de los Diputados en 1994. El diputado del Pozo, del Grupo Socialista resumía así las intervenciones: "acepto lo que todos los comparecientes casi unánimemente y los propios compañeros de la Comisión estamos constatando: que es imposible e indeseable intentar dar una definición de periodista". ' La rotundidad de la afirmación es buena prueba de la falta de claridad, el escaso consenso y la indefinición legal existentes en torno a esta cuestión. De hecho, acudir a otras fuentes no sirve sino para crear mayor confusión: así, el Diccionario de la Real Academia Española (2012) ofrece dos acepciones, que resultan restrictivas e incluso inexactas: ${ }^{2}$

1. Persona legalmente autorizada para ejercer el periodismo.

2. Persona profesionalmente dedicada en un periódico o en un medio audiovisual a tareas literarias o gráficas de información o de creación de opinión.

1 Entre las respuestas a la pregunta "¿Quién es periodista?", pueden destacarse las siguientes: "Problema gravísimo, definición muy confusa que ha quedado en manos de la empresa y que hay que dejar para más adelante" (Miguel Ángel Aguilar). "Cuestión espinosa en la que no entraré" (José Luís Gutiérrez); "Un tema enormemente complejo" (Marc Carrillo); o "No lo sé" (Inaki Gabilondo). El texto completo de las intervenciones aparece en los Diarios de Sesiones del Congreso de los Diputados español del 1 de junio, 22 de junio y 29 de noviembre de 1994. Un comentario puede verse en De Aguinaga, E. (2000). El periodista en el umbral del siglo XXI. En Sala de Prensa, 24. Consultado el 11 de junio de 2012. Disponible en http://www.saladeprensa.org/art157.htm.

2 Como señala Escobar Roca, G. (2002). Estatuto de los periodistas: régimen normativo de la profesión y organización de las empresas de comunicación. Madrid: Tecnos, p. 110, ambas definiciones son inexactas e incorrectas para la realidad española. La primera, "por ignorar palmariamente la realidad jurídica" (ya que en la práctica no se exige ninguna autorización). La segunda, por restringir los medios en los que el periodista puede desempeñar su actividad, olvidándose de Internet o las agencias informativas; y por introducir el elemento "creación de opinión", excluido en casi todas las definiciones y prohibido expresamente por varios códigos deontológicos. 
En ese sentido, es muy probable que el planteamiento de una consulta similar a la formulada en el Congreso de los Diputados en otros países provocaría una respuesta semejante. De hecho, las definiciones que ofrecen algunas instancias internacionales tampoco sirven para aclarar el panorama. Así, el Consejo de Europa (2000) considera periodista a "cualquier persona natural o legal que trabaja de manera regular o profesional recogiendo y divulgando información al público utilizando para ello cualquier medio de comunicación de masas". De nuevo aparece el término "profesional" y se evita la restricción en lo relativo al soporte mediante la fórmula "cualquier medio de comunicación de masas", pero la descripción del contenido resulta insuficiente, y tampoco hay referencias a los requisitos que debe cumplir el periodista, algo hasta cierto punto comprensible dada la variedad de ordenamientos existentes en los países de la Unión Europea.

Existen otras definiciones, más o menos exhaustivas, y una de las más completas es, posiblemente, la que ofrecen Nordenstreng y Topuz (1989) con el respaldo de la Organización Internacional de Periodistas (OIJ) y de la UNESCO:

... cualquier persona, de cualquier nacionalidad, que tenga un empleo fijo remunerado como director, reportero, fotógrafo, cameraman o técnico de prensa, radio, televisión o servicio de noticias filmadas, que ejerza su profesión cumpliendo normas ético-profesionales; tal actividad profesional consiste en buscar, recibir o impartir información, opiniones, ideas, estudios o comentarios en publicaciones diarias o periódicas, agencias de prensa, servicios de radiodifusión, televisión o cine.

En este caso se aporta un mayor número de elementos, como la dimensión ética; y otra característica básica para hablar de profesionalismo, el empleo fijo y remunerado. En cuanto al contenido de la tarea periodística, la descripción es más de- tallada, de manera que se trata de una definición más apropiada aunque carente todavía de ciertos componentes básicos, como la necesidad o no de formación previa.

En suma, ninguna de las propuestas existentes llega a aclarar con precisión si la actividad periodística puede considerarse o no una profesión, pues si bien en algunas ocasiones el término es utilizado, es evidente que de las características aportadas no puede deducirse tal realidad. Como ya se ha dicho, el debate viene de lejos y ha llenado miles de páginas. Por ello, el objetivo principal de este artículo será intentar caracterizar el periodismo en España de acuerdo con su situación actual para, a partir de ahí, intentar resolver la cuestión planteada. En ese sentido, dado que definir la actividad periodística es más que problemático, puede resulta útil desglosar, en primer lugar, brevemente el contenido del vocablo "profesión".

\section{La profesión. Definición y características}

El Diccionario de la Real Academia Española (2012) ofrece tres definiciones de profesión, de las que solo merece la pena considerar la tercera, por cuanto las dos anteriores hacen referencia a un sentido más religioso del término: "Empleo, facultad u oficio que alguien ejerce y por el que percibe una retribución".

Ese sentido religioso que menciona la Academia está muy presente en los orígenes del término y sobre todo en su sentido alemán, "beruf", que hace referencia a una misión encomendada por Dios, según ha puesto de manifiesto, entre otros, Max Weber (1979). Dicho componente ha desaparecido, en un contexto actual más secularizado, en la práctica totalidad de las profesiones, pero sí ha permanecido un componente moral que se traduce hoy en día en el servicio a la sociedad o al bien común por encima del propio, como puede observarse en la siguiente definición de Bell (1976): 
... actividad aprendida (es decir, escolarmente), lo que implica por tanto una preparación formal, pero dentro de un amplio contexto intelectual. Integrarse dentro de una profesión significa estar reconocido, formal o informalmente, por los colegas o por algún organismo establecido dentro de la profesión y una profesión engloba una norma de responsabilidad social. Lo que no quiere decir que los profesionales sean más benéficos o más magnánimos que sus compañeros sino que las expectativas sobre su conducta proceden de una ética de servicio que como norma, está por delante de una ética del interés propio. Por todas estas razones, la idea de una profesión implica las de competencia y autoridad, técnica y moral, y ocupación por el profesional de un puesto consagrado dentro de la sociedad.

En esta definición se aprecian ya varias de las que constituyen las características esenciales que toda actividad debe cumplir para ser considerada una profesión, como son la preparación formal, la existencia de un mecanismo interno de control, el sentido de la responsabilidad profesional, o la competencia técnica y moral. En todo caso, antes de continuar, conviene puntualizar que, como señalan, entre otros, Carr o Chadwick (2000), es posible definir el término de distintos modos según se tome en un sentido más amplio o más restringido:

1. Toda persona que recibe un salario por el trabajo realizado.

2. En otro sentido, cualquier trabajo bien hecho.

3. En su sentido más clásico y a la vez más exhaustivo, las actividades profesionales -bien realizadas o no- se diferencian de las ocupaciones por una serie de características y requerimientos necesarios para su ejercicio.

A este tercer concepto es al que hay que acudir para intentar responder al gran interro- gante de si el periodismo es o no una profesión. Para ello, se ofrece a continuación un elenco de características imprescindibles para poder decir con propiedad que una actividad constituye una profesión teniendo en cuenta que, como señala Hortal (1993):

... cuando todos esos rasgos se cumplen, estamos ante lo que podemos llamar la profesión en su sentido pleno, la profesión tipo. En cambio, cuando algunos de esos rasgos faltan o se cumplen en menor medida, no por ello hay que dejar de hablar de profesión, aunque sea en un sentido menos pleno.

Es decir, la profesionalización es un proceso dinámico por el que las diferentes actividades intentan alcanzar el ideal (profesionalismo en sentido pleno) que representan, por ejemplo, el Derecho o la Medicina. Las características que definen ese ideal son: ${ }^{3}$

1. Formación cualificada, teórica y práctica, por la que se transmite un saber sistemático y especializado. Dicha formación es conditio sine qua non para el acceso al ejercicio de la profesión.

3 Es evidente que las características aplicables a una definición estricta del término serían innumerables. Sin embargo, en un intento de sistematizar el análisis, se ha optado por acudir a una serie de autores que, o bien han estudiado la cuestión en relación con el mundo del periodismo, o bien han sido tomados como referencia para tratarla en el contexto periodístico, en especial Carr, D. (2000). Professional Education and Professional Ethics. En G. de Stexhe y J. Verstraeten (eds.). Matter of Breath: Foundations for Professional Ethics. Lovaina: Peeters; Davara, F. J. (1991). Profesionales de la comunicación. En A.Benito (dir.). Diccionario de Ciencias y Técnicas de la Comunicación. Madrid: Ediciones Paulinas; Dennis, E. E. y Merrill, J. (1996). Journalism as a profession. En E. E. Dennis (ed.). Media debates: issues in mass communication. White Plains, New York: Longman; Humanes, M.L. (1997). La formación de los periodistas en España. Tesis doctoral. Madrid: Facultad de Ciencias de la Información, Universidad Complutense; Hortal, A. (1993). Ética de las profesiones. Diálogo filosófico, 28, 205-222. 
2. Organización propia que regula internamente la profesión, en especial el acceso y el funcionamiento de la misma.

3. Dimensión ética que se formaliza en un código deontológico de conducta.

4. Alto grado de autonomía individual en el ejercicio de la actividad.

5. Campo específico de actividad, que cuenta con un cuerpo común de conocimientos, y que responde a una necesidad social.

6. Dedicación exclusiva, obteniendo a cambio una retribución.

A partir de aquí, se intentará contestar a la cuestión planteada, si el periodismo se puede o no considerar una profesión, aplicando para ello estos criterios. Los resultados obtenidos servirán, a su vez, para tratar de llegar al objetivo final, una definición del periodista.

\section{Formación cualificada imprescindible para ejercer la profesión}

El debate sobre si el futuro periodista debe contar con estudios previos es también largo en el tiempo y se articula en torno a dos posiciones antagónicas:

- El ejercicio del periodismo requiere la adquisición de una serie de conocimientos, teóricos y prácticos en un centro de enseñanza. El motivo principal es la complejidad de la sociedad actual y la gran responsabilidad que, en consecuencia, implica la labor periodística.

- El mejor escenario para aprender las técnicas necesarias para trabajar como periodista es la propia redacción. El periodismo es una actividad vocacional, relacionada con el talento natural. Cualquier tipo de regulación -y la exigencia de titulación o formación previa no deja de ser una más- supondría una amenaza contra la libertad de expresión.
Uno de las causas que explican esta polémica, que resultaría absurda para muchas otras actividades, puede encontrarse en el retraso que sufre el periodismo, no solo en su proceso de profesionalización, sino más concretamente en la puesta en marcha de sistemas formativos, cuya necesidad "ha aparecido tardíamente respecto a otras profesiones. Hasta el último tercio del siglo XIX la mayoría de los jóvenes escritores no habían recibido ningún tipo de formación y, según expresión de la época, habían asistido a la escuela de la vida" (Weaver, Drew y Wilhoit, 1991).

Por ello, muchas generaciones de periodistas no han considerado imprescindible cursar los programas educativos previos al desempeño de un puesto de trabajo, postura que ha ido perdiendo adeptos con el paso del tiempo y el progresivo aumento de titulados en las redacciones, aunque sin llegar a desaparecer totalmente, como lo demuestran, por ejemplo, las palabras de Gabriel García Márquez (1996), postulando que antes de la aparición de las facultades universitarias, se hacía un mejor periodismo, ya que "la misma práctica del oficio imponía la necesidad de formarse una base cultural, y el mismo ambiente de trabajo se encargaba de fomentarla. La lectura era una adicción laboral. Los autodidactos suelen ser ávidos y rápidos" y añadiendo que las facultades han creado "un pantano académico que satisface muchas de las necesidades actuales de la enseñanza, pero no son las que son propias del periodismo. Y menos las dos más importantes: la creatividad y la práctica".

A partir de estas dos posiciones iniciales, la realidad ofrece un panorama que difiere muchísimo de un país a otro, pero que puede sintetizarse en cuatro vías básicas de acceso al ejercicio de la profesión:

1. Los estudios universitarios de Periodismo o Comunicación.

2. La formación ofrecida por las escuelas profesionales. 
3. Los programas ofrecidos por los propios medios o por empresas relacionadas con el mundo de la comunicación.

4. El acceso totalmente libre sin que el proceso de formación esté sujeto a ningún tipo de regulación.

En el caso de España, la realidad muestra que la primera, la tercera y la cuarta vía son perfectamente válidas, mientras que, en consonancia con lo ocurrido en otras ocupaciones, no se han desarrollado programas específicos para periodistas en el ámbito de la formación profesional.

En este sentido, el Consejo de Europa (1993) parece pronunciarse a favor de la necesidad de la formación previa, aunque sin concretar los contenidos y el sistema más adecuados: "dada la complejidad del proceso informativo, basado cada vez más en la utilización de nuevas tecnologías, la rapidez y la síntesis, se debe exigir a los periodistas una formación profesional adecuada".

En palabras de Davara (1991), la formación previa diferencia al profesional del aficionado y el autodidacto, ya que el primero "debe tener una formación académica, completada después con el aprendizaje profesional. Nadie puede ser definido como profesional sin la formación adecuada. Otra cosa es cuál debe ser la formación académica que ha de recibir el profesional de la información".

En ese sentido, el carácter vocacional de la actividad periodística no está reñido, ni mucho menos, con la formación y el perfeccionamiento de la aptitud que, en el profesional, debe ser de dos tipos, como recuerda Todolí (1975): por un lado, natural, y por otro, dada o adquirida. La primera se corresponde con las preferencias e inclinaciones naturales de la persona; mientras que la segunda hace referencia a los años de formación, que dan lugar al dominio de un cuerpo de conocimientos.
Respecto a los contenidos de la enseñanza, cabe aventurar que la unificación de criterios, que muchas veces han situado a la universidad y a las escuelas profesionales en posiciones enfrentadas, podría servir para generalizar la necesidad de la formación y, por ende, avanzar en el camino hacia la profesionalización. En la medida en que el porcentaje de periodistas que cuente con estudios previos -universitarios o en escuelas profesionales pero específicos de su actividad- aumente, más cerca estará ese ideal.

\section{Organización profesional propia}

El acceso a la actividad periodística no está sometido actualmente en España a ningún tipo de requisito, ya sea la formación previa u otro cualquiera, y depende únicamente de lo que el empresario o director del medio correspondiente considere oportuno. Esa es la situación real del periodismo español, a pesar de que en 1984 se llegara, tras un largo debate, al acuerdo de establecer tres vías para poder ser inscrito en el Registro Profesional de Periodistas, que depende de la Federación de Asociaciones de la Prensa (FAPE): ser licenciado en Periodismo; ser licenciado en otra especialidad y contar con dos años de experiencia en medio de comunicación; o bien acreditar cinco años de trabajo como periodista.

Sin embargo, comoquiera que la FAPE no es un colegio profesional al uso, la inscripción en el Registro no es conditio sine qua non para trabajar en un medio y, en definitiva, no existe ningún tipo de normativa.

Lo mismo sucede en el ámbito de la Unión Europea: tampoco hay ninguna norma con rango legal que intente ofrecer una orientación, y de hecho, en la definición de "periodista" establecida por el Consejo de Europa no existe ninguna indicación en uno u otro sentido. Al respecto, como indica Bel Mallen (1992), "sería positivo la promulgación de una directiva que diese luz a esta cuestión y solucionase los posibles temas conflic- 
tivos que los distintos ordenamientos jurídicos europeos establecen".

En principio, esta medida ofrecería un mayor número de derechos y garantías para la actividad periodística. Sin embargo, las opiniones opuestas a esta posición argumentan que pondría en peligro, por un lado, la pluralidad de los medios de comunicación y, por otro, la libertad de expresión, debido a la necesaria intervención gubernamental. Así lo expresa el informe McBride (1980): "La experiencia demuestra que la concesión de licencias profesionales, así como todo procedimiento complicado de acreditación, tienden a fomentar la intervención del gobierno en la circulación nacional e internacional de la información".

En este sentido, es interesante recordar que los primeros intentos históricos de reglamentación profesional coincidieron con el surgimiento de los fascismos, en concreto con el ascenso al poder en Italia de Mussolini. Posteriormente, otros regímenes de este tipo, como España durante la dictadura franquista, tomaron medidas similares. Esto puede explicar el rechazo que genera en muchos sectores la sola idea de la reglamentación, vista como una posible vía de cortapisa gubernativa a la libertad de expresión. En todo caso, queda claro que en este punto el periodismo tampoco llega al nivel necesario como para hablar de profesionalismo. Más bien al contrario, todavía hoy en día, se trata de una actividad "marcada por el individualismo" (Ortega y Humanes, 2000).

\section{Dimensión ética y código de conducta}

La dimensión ética es un elemento básico del proceso de profesionalización, que se materializa en la elaboración de códigos deontológicos que definen las responsabilidades en el ejercicio de una actividad. A primera vista, este es uno de los puntos que más cerca se encuentran de cumplir los periodistas españoles, pues tanto la FAPE como varias de las respectivas asociaciones autonómicas cuentan con códigos de deontología periodística, de cuyo contenido se desprende que la dimensión ética y la actuación responsable se materializa, principalmente, en una actuación libre y honesta y en el respeto a la verdad.

Otra cuestión es la efectividad de estos documentos y si de su sola existencia puede desprenderse una verdadera actuación responsable de los periodistas. Como es sabido, el cumplimiento de este tipo de normas suele ser voluntario, por lo que, en última instancia, todo depende del grado de adhesión individual que susciten. Uno de los baremos básicos para comprobar si este es más o menos alto viene dado por las encuestas realizadas a periodistas en ejercicio, planteando las hipótesis de incumplir principios incluidos en los códigos. Pues bien, los datos (Weaver, 1998; Canel, Rodríguez y Sánchez Aranda, 2000) demuestran que el consenso entre los periodistas españoles sobre las pautas básicas de comportamiento ético está "entre los más altos del mundo". En conclusión cabe hablar de un mayor acercamiento al ideal del profesionalismo en este punto frente a los dos anteriores.

\section{Alto grado de autonomía individual}

La gran responsabilidad que encierra el trabajo del periodista es la principal razón que le obliga, al menos moralmente, a ser él mismo quien defina en todo momento las pautas de su labor informativa. Sin embargo, su capacidad de decisión individual depende, como es sabido, de otros muchos factores que entran en juego, por lo que ha sido puesta en entredicho a menudo.

La permanente sospecha de que es la propia empresa informativa -cuando no otros intereses en un nivel superior- la que prima sobre la propia voz del redactor individual a la hora de establecer los criterios o, en último término, publicar o no publicar, es inevitable. Al respecto, Tuchman (1983) es contundente: "el profesionalismo entre los periodistas es saber cómo lograr un relato que satisfaga las necesidades y las pautas de la organización". 
Cabe señalar que dos son las armas legales que maneja el periodista para preservar, al menos en cierto grado, su autonomía. Por un lado, la cláusula de conciencia, que le permite negarse a desempeñar una tarea o incluso, llegado el caso, a abandonar su empresa con la indemnización correspondiente, si dicha tarea es contraria a sus convicciones morales. Por otro, el secreto profesional, derecho a la vez que deber, que protege al propio periodista al facilitar el acceso a las fuentes. Por tanto, puede decirse que el grado de autonomía de un periodista dependerá, en buena medida, de cómo regule estos dos aspectos el ordenamiento jurídico correspondiente.

De todos modos, la eficacia de ambas figuras, y de la cláusula de conciencia en particular, hay que acogerla con cautela, ya que abandonar la empresa, en el caso de periodistas sin un cierto renombre, puede traer como consecuencia inevitable el desempleo, con lo que la opción tomada en muchos casos es plegarse ante los intereses empresariales. En este sentido, valgan los datos de la encuesta realizada hace unos años por la Asociación de la Prensa de Madrid y recogida por la revista Periodistas (1990). Ante la afirmación: "Los periodistas, igual que los lectores están indefensos ante la manipulación de la información de las empresas", el 71'9\% de los encuestados se mostró de acuerdo mientras que solo el 20'6\% manifestó su desacuerdo.

\section{Campo específico de actividad y servicio a la sociedad}

Del hecho de que resulte tan difícil definir el periodismo se desprende que intentar identificar una serie de conocimientos específicos es igual de complicado. Más allá de que, como característica común a todos los que lo ejercen, el periodismo es una actividad necesaria, ya que responde a un bien social fundamental, como es el derecho a la información de todos los ciudadanos, poco más se puede decir en este sentido. Así, para Muñoz Alonso, el periodismo es peculiar precisamente "por el tipo de conocimientos que exige para su ejercicio. El periodismo, más allá de unas reglas básicas de carácter general, no tiene fronteras. No es como una profesión "clásica", donde existe un acervo de conocimientos y técnicas bien definido" (recogido por Escobar Roca, 2002).

Por otro lado, en la definición de Nordenstreng y Topuz antes mencionada puede verse la gran variedad de tipologías que pueden englo barse bajo el epígrafe "periodista". Una relación que se hace mucho menos clara en el período de convergencia mediática y en la era digital actual: las tradicionales líneas divisorias entre prensa escrita, radio y televisión se han visto alteradas con la aparición de Internet y las distintas aplicaciones multimedia. En un primer nivel, el abanico de habilidades requeridas hasta ahora para trabajar como periodista se amplía enormemente. En un segundo nivel, se plantean nuevos interrogantes sobre el perfil del periodista, y, en concreto, si puede considerarse como tal la persona que coloca información en la red de manera independiente, sin estar empleado por ningún tipo de organización periodística y sin poseer ningún tipo de formación previa. Algunos autores han intentado precisar un elenco básico de conocimientos necesarios para el periodista. Meyer (1991) habla de "cómo encontrar información, cómo evaluarla y analizarla, y por último, cómo comunicarla de manera idónea y adecuada a las necesidades del público". Sin embargo, probablemente bastaría con plantear si ese proceso de evaluación y análisis de la información exige o no una formación previa de tipo general, para volver a crear la controversia.

Finalmente, otro inconveniente es la atípica relación profesional-cliente que se establece en el caso del periodista, que cuenta con una doble clientela, los publicitarios y la audiencia. El ejercicio del periodismo parte del supuesto de que el servicio se ofrece a toda la sociedad (de ahí expresiones como servicio público) pero no puede decirse que, en general, exista una relación directa entre el periodista y el público, algo para lo que, 
no obstante, Internet y las nuevas tecnologías ofrecen una ocasión incomparable para llegar a la deseada interactividad.

\section{Dedicación exclusiva}

En este último punto, basta con referirse a los numerosos estudios realizados, que hablan, en general, de precariedad laboral, especialmente entre los más jóvenes, y de un gran contraste entre un pequeño grupo de periodistas de renombre y entre el grupo más numeroso, cuyos sueldos y estabilidad no suelen ser demasiado altos, lo que convierte el pluriempleo en un recurso fácil. ${ }^{4}$

\section{El periodismo todavía no es una profesión}

Una vez analizadas las características constitutivas de una profesión, difícilmente se puede llegar a la conclusión de que el Periodismo ha alcanzado tal estatus "en sentido pleno". Es cierto que con una aplicación estricta ni siquiera las profesiones clásicas lo harían, entre otras cosas, por el simple hecho de que en cualquier actividad hay buenos y malos profesionales. Más allá de esa salvedad las diferencias con la Medicina y el Derecho son evidentes. El Periodismo no ha seguido el camino más usual que el resto de ocupaciones han emprendido hacia la consecución de este nivel, y cuando lo ha hecho, ha sido con retraso.

A la hora de buscar causas y/o causantes de esta realidad, muchas son las posibles respuestas. Algunos han señalado a la empresa periodística como la principal responsable, pues ello le permite contratar a trabajadores con menos derechos. Sin embargo, no hay que excluir a los propios periodistas que, quizá por las nefastas consecuencias derivadas de algunos intentos estatales de regla-

$4 \mathrm{Al}$ respecto, entre los numerosísimos estudios, como ejemplo ilustrativo del caso español pueden verse los datos que refleja el estudio de Canel, M.J.; Rodríguez, R. y Sánchez-Aranda, J. J. (2000). Periodistas al descubierto. Retrato de los profesionales de la información. Madrid: Centro de Investigaciones Sociológicas. mentación bajo sistemas dictatoriales, han contribuido a aumentar la ambigüedad que envuelve a su actividad "al interpretar que su trabajo no está sometido a reglas -al margen de las que en cada momento decida el propio grupo de profesionalesy que cualquier intento de institucionalización de la actividad periodística atenta contra la libertad de expresión" (Humanes, 1997).

En este sentido, son significativas las palabras de Merrill en su debate con Dennis (1997):

El periodismo no es una profesión y además no debe serlo. El periodismo es una ocupación, un oficio, un compromiso abierto a todo el mundo, con independencia de su nivel de estudios. Cuanto más cerca esté el periodismo de alcanzar el estatus de profesión, [...] más limitado estará el pluralismo y los periodistas no pensarán en el interés público sino tendrán más presentes sus propios intereses.

Por lo tanto, el debate continúa abierto y seguramente lo seguirá estando durante mucho tiempo. Ante esta ambigua situación, otros autores optan por una decisión salomónica. Así, Bromley (1991) señala que "en sentido estricto, el periodismo no es ni una profesión ni un oficio, por cuanto posee características propias de ambos". Por su parte, Lambeth (1992) plantea la definición de periodismo como "oficio dotado de responsabilidad profesional". Sin embargo, de lo que no cabe duda es que el periodismo es una ocupación cada vez más profesionalizada y que, a medida que este proceso avance, y que la actividad periodística logre un mayor reconocimiento, ello repercutirá sin duda en un mejor desempeño de sus actores, los periodistas.

\section{Conclusión. Hacia un intento de definición del periodista ideal}

Como conclusión, tratando de responder a la pregunta formulada hace unos años en el Congreso de los Diputados español, se podría 
ofrecer una definición que, más que adecuarse a la realidad, se englobaría en una especie de figura del periodista ideal:

... profesional (en sentido amplio) que, tras haber recibido una formación previa teórico práctica, que conjuga los elementos generales con la especialización, en una institución educativa acreditada para ello, ejerce el Periodismo como puesto de trabajo único, recibiendo a cambio una retribución, y cuya misión consiste en la elaboración y transmisión a la sociedad de informaciones, siguiendo para ello los criterios éticos materializados en los códigos deontológicos.

\section{Referencias bibliográficas}

De Aguinaga, Enrique (2000). El periodista en el umbral del siglo XXI. En Sala de Prensa, 24. Consultado el 11 de junio de 2012. Disponible en http://www. saladeprensa.org/art157.htm.

Asamblea Parlamentaria del Consejo de Europa (1993). Código Europeo de Deontología del Periodismo. Estrasburgo, 1 de julio. Resolución aprobada por unanimidad. Ponente y redactor: Manuel Núñez Encabo.

Bel Mallen, I. (1992). Teoría general del sujeto profesional. En I. Bel Mallen, L. Corredoira y P. Cousido (eds.) Derecho de la información (I) Sujetos y medios. Madrid: Colex.

Bell, D. (1976). El advenimiento de la sociedad industrial. Madrid: Alianza.

Bromley, M. (1997). The End of Journalism? Changes in Workplace in the Press and Broadcasting in the 1990s. En M. Bromley y T. O'Malley (eds.). A journalism reader. London: Routledge.

Canel, M. J.; Rodríguez, R. y Sánchez-Aranda, J. J. (2000). Periodistas al descubierto. Retrato de los profesionales de la información. Madrid: Centro de Investigaciones Sociológicas.
Carr, D. (2000). Professional Education and Professional Ethics. En G. de Stexhe y J. Verstraeten (eds.). Matter of Breath: Foundations for Professional Ethics. Lovaina: Peeters.

Chadwick, R. (2000). Professional Ethics. En G. de Stexhe y J. Verstraeten (eds.). Matter of Breath: Foundations for Professional Ethics. Lovaina: Peeters.

Comité de Ministros del Consejo de Europa (2000). Apéndice a la Recomendación n. R(2000)7 a los Estados miembros sobre el derecho de los periodistas a no revelar sus fuentes de información. 8 de marzo.

Davara, F. J. (1991). Profesionales de la comunicación. En Ángel Benito (dir.). Diccionario de Ciencias y Técnicas de la Comunicación. Madrid: Ediciones Paulinas

Dennis, E. E. y Merrill, J. (1996). Journalism as a profession. En E. E. Dennis (ed.). Media debates: issues in mass communication. White Plain, New York: Longman.

Escobar Roca, G. (2002). Estatuto de los periodistas: régimen normativo de la profesión y organización de las empresas de comunicación. Madrid: Tecnos.

García Márquez, G. (1996). El mejor oficio del mundo. Discurso pronunciado ante la $52^{\mathrm{a}}$ Asamblea de la Sociedad Interamericana de Prensa (SIP). Los Ángeles, 7 de octubre.

Hortal, A. (1993). Ética de las profesiones. Diálogo filosófico, 28, 205-222.

Humanes, M. L. (1997). La formación de los periodistas en España. Tesis doctoral. Madrid: Facultad de Ciencias de la Información. Universidad Complutense.

Lambeth, E. (1992). Committed journalism: an ethic for the profession. Bloomington: Indiana University Press.

McBride, S. (dir.) (1980). Un solo mundo, voces múltiples: comunicación e información en nuestro tiempo. México: Fondo de Cultura Económica.

Meyer, P. (1991). The New Precision Journalism. Bloomington: Indiana University Press. 
Nordenstreng, K. y Topuz, H. (1989). Journalist: Status, Rights and Responsibilities. Prague: International Organization of Journalists.

Ortega. F. y Humanes, M. L. (2000). Algo más que periodistas. Sociología de una profesión. Madrid: Ariel Sociología.

Periodistas (1990): El periodista español. Retrato intermitente, $n^{\circ} 39$, diciembre.

Real Academia Española (2012). Diccionario de la lengua española. Consultado el 12 de junio de 2012. Disponible en http://www.rae.es/rae.html

Todolí Duque, J. (1975). Nivel ético del profesional español. Madrid: Confederación Española de Cajas de Ahorro.
Tuchman, G. (1983): La producción de la noticia: estudio sobre la construcción de la realidad. Barcelona: Gustavo Gili.

Weaver, D. (ed.) (1998). The Global Journalist. New Jersey: Hampton Press.

Weaver, D. H., Drew D. y Wilhoit, G.C. (1991) U.S. Television, Radio and Newspaper Journalists. En Journalism Quarterly, 63, 683-692

Weber, M. (1979). La ética protestante y el espíritu del capitalismo. Barcelona: Península. 